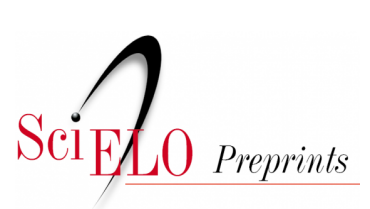

Situação: O preprint foi submetido para publicação em um periódico

\title{
Mortalidade e morbidade em crianças e adolescentes por COVID-19 no Brasil
}

Leonor Maria Pacheco Santos, Erly Catarina Moura, Luciana Gonzaga Oliveira, Fabrício Vieira

Cavalcante, Klébya Hellen Dantas Oliveira, Geraldo Magela Fernandes, Ivana Cristina de Holanda Cunha Barreto

https://doi.org/10.1590/SciELOPreprints.2069

Este preprint foi submetido sob as seguintes condições:

- O autor submissor declara que todos os autores responsáveis pela elaboração do manuscrito concordam com este depósito.

- Os autores declaram que estão cientes que são os únicos responsáveis pelo conteúdo do preprint e que o depósito no SciELO Preprints não significa nenhum compromisso de parte do SciELO, exceto sua preservação e disseminação.

- Os autores declaram que a pesquisa que deu origem ao manuscrito seguiu as boas práticas éticas e que as necessárias aprovações de comitês de ética de pesquisa estão descritas no manuscrito, quando aplicável.

- Os autores declaram que os necessários Termos de Consentimento Livre e Esclarecido de participantes ou pacientes na pesquisa foram obtidos e estão descritos no manuscrito, quando aplicável.

- Os autores declaram que a elaboração do manuscrito seguiu as normas éticas de comunicação científica.

- Os autores declaram que o manuscrito não foi depositado e/ou disponibilizado previamente em outro servidor de preprints ou publicado em um periódico.

- O autor submissor declara que as contribuições de todos os autores estão incluídas no manuscrito.

- O manuscrito depositado está no formato PDF.

- Os autores declaram que caso o manuscrito venha a ser postado no servidor SciELO Preprints, o mesmo estará disponível sob licença Creative Commons CC-BY.

- Caso o manuscrito esteja em processo de avaliação ou sendo preparado para publicação mas ainda não publicado por um periódico, os autores declaram que receberam autorização do periódico para realizar este depósito.

Submetido em (AAAA-MM-DD): 2021-03-31

Postado em (AAAA-MM-DD): 2021-04-16 
COVID-19 e SIM-P: morbimortalidade em crianças e adolescentes no Brasil, 2020-2021

COVID-19 and MIS-C:morbidity and mortality in children and adolescents in Brazil, 2020-2021

Leonor Maria Pacheco Santos, leopac@unb.br, https://orcid.org/0000-0002-6739-6260,

Departamento de Saúde Coletiva, Universidade de Brasília, Brasília, Distrito Federal, Brasil. CEP 70910-900

Erly Catarina de Moura, erlycm@gmail.com, http://orcid.org/0000-0002-9237-432X, Departamento de Saúde Coletiva, Universidade de Brasília, Brasília, Distrito Federal, Brasil. CEP 70910-900

Luciana Gonzaga de Oliveira, lucianagoliveira7@ gmail.com. https://orcid.org/0000-00032399-3052), Departamento de Saúde Coletiva, Universidade de Brasília, Brasília, Distrito Federal, Brasil. CEP 70910-900

Fabrício Vieira Cavalcante, fabricioocavalcante@ gmail.com, https://orcid.org/0000-00028706-0457), Departamento de Saúde Coletiva, Universidade de Brasília, Brasília, Distrito Federal, Brasil. CEP 70910-900

Klébya Hellen Dantas de Oliveira, oliveirakhd@gmail.com, http://orcid.org/0000-00023600-4009, Departamento de Saúde Coletiva, Universidade de Brasília, Brasília, Distrito Federal, Brasil. CEP 70910-900

Geraldo Magela Fernandes, geraldomafer@gmail.com, https://orcid.org/0000-0002-43478886, Área de Medicina da Criança e Adolescente, Faculdade de Medicina, Universidade de Brasília, Brasília, Distrito Federal, Brasil. CEP 70910-900

Ivana Cristina de Holanda Cunha Barreto, ivana.barreto24@ gmail.com, http://orcid.org/00000001-8447-3654, Fundação Oswaldo Cruz Ceará, Eusébio, Ceará, Brasil. CEP 61760-000

Contribuição dos autores: todos os autores participaram da concepção, estruturação, análise e interpretação dos dados e aprovaram a versão final do manuscrito

Financiamento: Chamada Pública MCTI/CNPq/CT-Saúde/MS/SCTIE/Decit No 07/2020 


\section{RESUMO}

Objetivos: descrever a evolução temporal da morbidade e mortalidade por COVID-19 e síndrome inflamatória multissistêmica pediátrica temporalmente associada à COVID-19, a SIM-P, em crianças e adolescentes brasileiros.

Métodos: trata-se de estudo descritivo, baseado em dados secundários oficiais. A mortalidade por COVID-19 foi analisada de acordo com os dados do Portal da Transparência do Registro Civil. A morbimortalidade por SIM-P foi verificada a partir de boletins epidemiológicos estaduais e federal.

Resultados: no período de um ano registraram-se 2.346 óbitos por COVID-19 em crianças e adolescente. Do total, $78 \%$ são óbitos entre adolescentes, que vem aumentando exponencialmente em 2021, na segunda onda da epidemia. A taxa de mortalidade por COVID19 nas crianças foi de 1,8 por 100 mil habitantes, enquanto em adolescentes foi três vezes maior (5,6 por 100 mil habitantes). A SIM-P foi notificada em 26 das 27 Unidade da Federação e $77 \%$ dos casos incidem em menores de 10 anos.

Conclusões: o aumento da morbimortalidade por COVID-19 em crianças e adolescentes reforça a necessidade de medidas drásticas de contenção da epidemia. A COVID-19 apresenta consequências a curto e longo prazo, podendo comprometer a saúde de crianças e adolescentes, além de interferir no seu desenvolvimento integral, atividade física, socialização adequada, desempenho escolar e, futuramente, na sua inserção plena na sociedade.

Palavras-chave: COVID-19. Criança. Adolescente. Morbimortalidade. Brasil. 


\begin{abstract}
Objectives: to describe the temporal evolution of morbidity and mortality due to COVID-19 and pediatric multisystemic inflammatory syndrome temporally associated with COVID-19, MIS-C, in Brazilian children and adolescents.

Methods: This is a descriptive study, based on official secondary data. Mortality due to COVID19 was analyzed according to data from the Civil Registry Transparency Portal. MIS-C morbidity and mortality was verified using state and federal epidemiological bulletins.

Results: within a year 2,346 deaths from COVID-19 were recorded in children and adolescent. From this total $78 \%$ are deaths among adolescents, which has been increasing exponentially in 2021, in the second wave of the epidemic. The mortality rate due to COVID-19 in children was 1.8 per 100 thousand inhabitants, while in adolescents it was three times higher (5.6 per 100 thousand inhabitants). MIS-C was notified in 26 of the 27 Federation Units and $77 \%$ of the cases are in children under 10 years old.

Conclusions: the increase in COVID-19 morbidity and mortality in children and adolescents reinforces the need for drastic measures to contain the epidemic. COVID-19 has short- and long-term consequences, which can compromise the health of children and adolescents, in addition to interfering in their integral development, physical activity, adequate socialization, school performance and, in the future, their full insertion in society
\end{abstract}

Keywords: COVID-19. Child. Adolescent. Mortality. Morbidity Brazil. 


\section{INTRODUÇÃO}

Em janeiro de 2020 a Organização Mundial da Saúde (OMS) declarou a Emergência de Saúde Pública de Importância Internacional por surto de novo coronavírus. Um ano depois, no Brasil, mais de 12 milhões de casos confirmados e 340 mil mortes por COVID-19 sinalizam a gravidade da epidemia ${ }^{1}$.

Idosos e indivíduos com doenças crônicas não transmissíveis são considerados os principais grupos de risco para COVID-19. No entanto, a doença acomete indivíduos de todas as faixas etárias, tendo sido observado o aumento da incidência na população pediátrica, especialmente entre adolescentes ${ }^{2}$. Ainda que com manifestações clínicas mais leves e menor risco de complicações $^{3}$, a população pediátrica pode evoluir ao óbito por COVID-19 ${ }^{4}$.

Além disto, uma complicação rara da infecção pelo SARS-CoV-2, a síndrome inflamatória multissistêmica pediátrica temporalmente associada à COVID-19 (SIM-P) ${ }^{\mathbf{5}}$, tem sido descrita nesta população, como um quadro clínico complexo e potencialmente grave, podendo também levar ao óbito. A SIM-P costuma acometer crianças maiores de 5 anos de idade e a hipótese de uma possível associação temporal com a infecção pelo SARS-COV-2 foi aventada, visto que as crianças afetadas testaram positivas para o vírus ${ }^{\mathbf{5}}$. O início dos sintomas tem sido relatado entre quatro a seis semanas após diagnóstico de COVID-19. Caso de SIM-P associada à COVID-19 é definido pela OMS como um quadro febril, com aumento dos marcadores inflamatórios por três ou mais dias, associado com diagnóstico prévio de COVID-19 ou contato com casos confirmados, acompanhados de duas das seguintes manifestações, excluídas outras causas infecciosas para o quadro inflamatório: i) erupção cutânea ou conjuntivite não purulenta bilateral ou sinais de inflamação mucocutânea (oral, mãos ou pés); ii) hipotensão ou choque; iii) características de disfunção miocárdica, pericardite, valvulite ou anormalidades coronárias (incluindo achados de ecocardiograma ou troponina elevada ou peptídeo natriurético Nterminal pro tipo B); iv) evidência de coagulopatia (tempo de protrombina elevado, tempo de 
tromboplastina parcial e dímeros $\mathrm{D}$ elevados), e; v) problemas gastrointestinais agudos (diarreia, vômito ou dor abdominal) ${ }^{\mathbf{6}}$.

Frente ao aumento do número de casos de COVID-19 em crianças e adolescentes e o surgimento da SIM-P, o objetivo deste estudo é descrever a morbimortalidade por COVID-19 em indivíduos de 0 a 19 anos no Brasil.

\section{MÉTODOS}

Trata-se de estudo descritivo, baseado em dados secundários oficiais. A mortalidade por COVID-19 foi analisada de acordo com os dados do Portal da Transparência do Registro Civil e a morbimortalidade por SIM-P foi verificada a partir de boletins epidemiológicos estaduais e federal.

No Brasil, o Conselho Nacional de Justiça mantém o Portal da Transparência do Registro Civil ${ }^{\mathbf{1}}$, que permite o acesso a dados sobre nascimentos, casamentos e óbitos, obtidos dos cartórios de Registro Civil, segundo a Unidade da Federação (UF). Em 2020, o Portal passou a divulgar os óbitos por COVID-19 com atualização diária. Para este estudo, o número de óbitos mensal de crianças (< 10 anos) e adolescentes (10 a 19 anos de idade) foi extraído para o período de 01/03/2020 a 31/03/2021. A taxa de mortalidade por COVID-19 em crianças e adolescentes, foi calculada a cada 100 mil habitantes para UF e o Brasil.

Após a identificação da SIM-P temporalmente associada à COVID-19 ${ }^{\mathbf{5 , 6}}$ o Ministério da Saúde implantou o monitoramento nacional a partir de 24/07/2020; os casos de SIM-P ocorridos antes desta data foram notificados de forma retroativa. O número cumulativo de casos confirmados e óbitos por SIM-P, segundo UF de notificação, foi obtido do Boletim Epidemiológico do Ministério da Saúde (volume 52, número 12), incluindo dados até março de 2021 e de boletins epidemiológicos estaduais, atualizados até a mesma data. A incidência de casos confirmados de SIM-P foi calculada por 100 mil habitantes. 
As taxas de mortalidade e a incidência foram calculadas utilizando a estimativa populacional do Instituto Brasileiro de Geografia e Estatística para as faixas etárias analisadas, no ano $2020^{7}$.

\section{RESULTADOS}

Foram registrados 2.346 óbitos por COVID-19, sendo 521 (22\%) em crianças e 1.825 (78\%) em adolescentes. A Figura 1 mostra que a curva epidêmica de óbitos por COVID-19 em adolescentes apresenta duas ondas, a primeira com pico em maio de 2020, que declina até novembro e recrudescimento a partir de dezembro de 2020. Apesar do aumento exponencial no número de mortes de adolescentes na segunda onda, o ápice pode ainda não ter sido atingido. Entre as crianças, o número de óbitos foi maior em maio de 2020, não sendo evidente uma segunda onda nesta faixa etária.

A taxa de mortalidade por COVID-19 em crianças foi de 1,8 por 100 mil habitantes (Figura 2), enquanto em adolescentes foi três vezes maior (5,6 por 100 mil habitantes). Roraima, Acre, Sergipe e Rondônia foram as UF com as maiores taxas de mortalidade por COVID-19 em crianças. Entre os adolescentes, as maiores taxas de mortalidade ocorreram no Acre, Roraima, Ceará e Sergipe. Em contrapartida, as menores taxas de mortalidade entre crianças foram observadas no Piauí, Amapá e Distrito Federal; e entre adolescentes no Piauí, Minas Gerais, Mato Grosso do Sul e Maranhão. Tocantins não registrou nenhum óbito por COVID-19 em crianças.

Em agosto de 2020 ocorreram as primeiras notificações de SIM-P. Em apenas 22 dias foram relatados 197 casos no Boletim Epidemiológico 51, número 35, que, provavelmente, representam casos represados desde o início da pandemia. Nos sete meses seguintes, de setembro de 2020 a março de 2021, a média mensal foi em torno de 89 casos novos.

De acordo com a Tabela 1, foram confirmados 822 casos e 52 óbitos por SIM-P até março de 2021. A incidência da SIM-P foi maior em crianças, que correspondem a 77,1\% dos casos. São 
Paulo e Minas Gerais, as UF mais populosas, apresentaram as maiores ocorrências de morbimortalidade. Entretanto, a maior incidência de casos foi observada no Distrito Federal.

\section{DISCUSSÃO}

Este estudo mostrou que enquanto adolescentes tem apresentado maior número de óbitos por COVID-19, a SIM-P tem acometido mais as crianças. Parte da população pediátrica é assintomática à infecção pela COVID-19 e apresenta manifestações clínicas leves; mas o aumento de morte em adolescentes e a morbimortalidade da SIM-P é preocupante.

Há algumas razões que podem justificar a disparidade na apresentação clínica entre os quadros pediátricos e adultos. Uma delas é a diferença na atividade da enzima conversora de angiotensina nestas populações. Outra possibilidade está relacionada à imunidade na população pediátrica, com uma concentração maior de células CD4 quando comparadas aos adultos ${ }^{8}$. No entanto, achados deste estudo reforçam a necessidade de proteger crianças e adolescentes da COVID-19.

A SIM-P é caracterizada por uma resposta inflamatória do organismo que atinge vários sistemas, com características similares a outras síndromes que atingem crianças e adolescentes 9 tais como síndrome de Kawasaki e o choque séptico ${ }^{\mathbf{1 0}}$. Todavia, apresenta pouco acometimento pulmonar, uma das principais características da COVID-19 em adultos ${ }^{11}$. Os fatores de risco para desenvolvimento da SIM-P incluem a idade, a carga viral e as comorbidades crônicas ${ }^{2}$. Há dificuldades no manejo clínico pois o tratamento para SIM-P ainda não foi completamente estabelecido $^{\mathbf{1 2}}$. Ensaios clínicos são necessários para definir qual o tratamento ideal para a reversão da resposta inflamatória, a prevenção de aneurismas de artérias coronárias e a mortalidade na população afetada ${ }^{\mathbf{1 0}}$. Recomenda-se o desenvolvimento de estratégias de qualificação profissional para o diagnóstico e assistência de casos de SIM-P no Brasil ${ }^{\mathbf{1 3}}$.

Tanto a COVID-19 quanto a SIM-P tem atingido crianças e adolescentes em todo o Brasil. Comparando o diferencial da mortalidade entre estes grupos, uma hipótese é a possibilidade de 
maior confinamento das crianças. As medidas de proteção, mitigação e contenção da COVID19 têm se mostrado eficazes; assim, o confinamento residencial, o distanciamento social em situações públicas necessárias e o uso de máscaras por crianças a partir de cinco anos é fundamental ${ }^{14}$.

Além dos efeitos clínicos diretos, efeitos indiretos da COVID-19 na saúde das crianças e adolescentes incluem prejuízos no ensino, socialização e desenvolvimento; afastamento do convívio familiar ampliado, amigos e rede de apoio influindo na saúde mental; inatividade física; uso excessivo de mídias, telas, smartphones; desafios para garantia da vacinação e atendimento de puericultura; aumento da insegurança alimentar e fome $\mathrm{e}^{\mathbf{1 3}}$.

No que se refere à educação formal, há divergências sobre a manutenção ou o retorno das aulas presenciais. Entretanto, estes resultados reforçam que a paralisação de atividades presenciais deve ser considerada, em todos os grupos etários, principalmente para evitar o encontro e aglomeração de jovens ${ }^{15}$.

Os achados sugerem um aumento da morbimortalidade em crianças e adolescente, que podem ser ainda maiores, considerando as limitações inerentes aos bancos de dados disponíveis, que são sujeitos a atrasos de notificação, subnotificação e, ainda, algumas imprecisões diagnósticas.

\section{CONCLUSÕES}

Os dados aqui apresentados são consistentes com a evolução da pandemia no país e alertam para a importância de continuar investindo em ações de mitigação e contenção da COVID-19. Estima-se que atualmente cerca de $29 \%$ da população brasileira esteja abaixo de 20 anos de idade, o que impõe a necessidade de medidas urgentes e radicais no sentido de proteger a população mais jovem dos seus efeitos negativos. A morbimortalidade por COVID-19 tem consequências a curto e longo prazo, podendo comprometer a saúde de crianças e adolescentes, interferindo no seu desenvolvimento integral, na socialização adequada, no desempenho escolar e, futuramente, na sua inserção plena na sociedade. 


\section{REFERÊNCIAS}

1. Associação dos Registradores de Pessoas Naturais. Portal da Transparência - Registro Civil. [Internet] Accessed 26 Mar. 2021: https://transparencia.registrocivil.org.br/especialcovid\#: :text=A\%20fam\%C3\%ADlia\%20tem\%20at\%C3\%A9\%2024h,)\%2C\%20que\%20atu aliza\%20esta\%20plataforma.

2. Dong Y, Mo X, Hu Y, Qi X, Jiang F, Jiang Z et al. Epidemiology of COVID-19 among children in China. Pediatrics. 2020; 145(6):e20200702.

3. Pavone P, Ceccarelli M, Taibi R, Rocca GL, Nunnari G. Outbreak of COVID-19 infection in children: fear and serenity. Eur Rev Med Pharmacol Sci 2020; 24:(8)4572-4575.

4. Hillesheim D, Tomasi YT, Figueiró TH. Síndrome respiratória aguda grave por COVID-19 em crianças e adolescentes no Brasil: perfil dos óbitos e letalidade hospitalar até a 38a Semana Epidemiológica de 2020. Epidemiol Serv Saude, 2020; 29(5):e2020-644.

5. Radia T, Williams N, Agrawal P, Harman K, Weale J, Cook J, et al. Multi-system inflammatory syndrome in children \& adolescents (MIS-C): A systematic review of clinical features and presentation. Paediatric Respir Rev 2020; 11:S1526-0542(20)30117-2.

6. WHO. Multisystem inflammatory syndrome in children and adolescents with COVID19. Accessed Apr. 7, 2021 https://www.who.int/news-room/commentaries/detail/multisysteminflammatory-syndrome-in-children-and-adolescents-with-covid-19

7. Brasil. Projeção da população por Unidades da Federação por sexo e idade para o período 20002030. Accessed 2 Apr. 2021: http://tabnet.datasus.gov.br/cgi/tabcgi.exe?ibge/cnv/projpopuf.def

8. Zhu, L., Lu, X. \& Chen, L. Possible causes for decreased susceptibility of children to coronavirus. Pediatr Res 88, 342 (2020). https://doi.org/10.1038/s41390-020-0892-8

9. Sociedade Brasileira de Pediatria. Síndrome inflamatória multissistêmica em crianças e adolescentes provavelmente associada à COVID-19: uma apresentação aguda, grave e potencialmente fatal. Nota de Alerta 2020. [internet]. Accessed Apr. 7, 2021 
https://www.sbp.com.br/fileadmin/user_upload/22532d-

NA_Sindr_Inflamat_Multissistemica_associada_COVID19.pdf.

10. Jiang L, Tang K, Levin M, Irfan O, Morris SK, Wilson K, Klein JD, Bhutta ZA. COVID-19 and multisystem inflammatory syndrome in children and adolescents. Lancet Infect Dis. 2020; Nov;20(11):e276-e288. doi: 10.1016/S1473-3099(20)30651-4.

11. Weisberg SP Connors T, Zhu Y, Baldwin M, Lin WH, Wontakal S et al. Antibody responses to SARS-CoV2 are distinct in children with MIS-C compared to adults with COVID19. medRxiv, 2020; Jul 14:2020.07.12.20151068 [Preprint].

12. Levin M. Childhood multisystem inflammatory syndrome - a new challenge in the pandemic. N Engl J Med, 2020; 383(4):393-5.

13. Instituto Fernandes Figueira. COVID-19 e saúde da criança e do adolescente. Rio de Janeiro: Fiocruz, 2020. Accessed 27 Mar. 2021. http://www.iff.fiocruz.br/pdf/covid19_saude_crianca_adolescente.pdf

14. Souza ASR et al. Aspectos gerais da pandemia de COVID-19. Rev. Bras. Saúde Matern. Infant., Recife, 2021; 21(Supl. 1):S47-S64. Accessed 2 abr. 2021. https://www.scielo.br/pdf/rbsmi/v21s1/pt_1519-3829-rbsmi-21-s1-0029.pdf

15. Gutiérrez AC. Contribuições para o retorno às atividades escolares presenciais no contexto da pandemia Covid-19. Rio de Janeiro: Fiocruz, 2020. Accessed 27 Mar. 2021: https://portal.fiocruz.br/sites/portal.fiocruz.br/files/documentos/contribuicoes_para_o_retorno _escolar_-08.09_4_1.pdf 


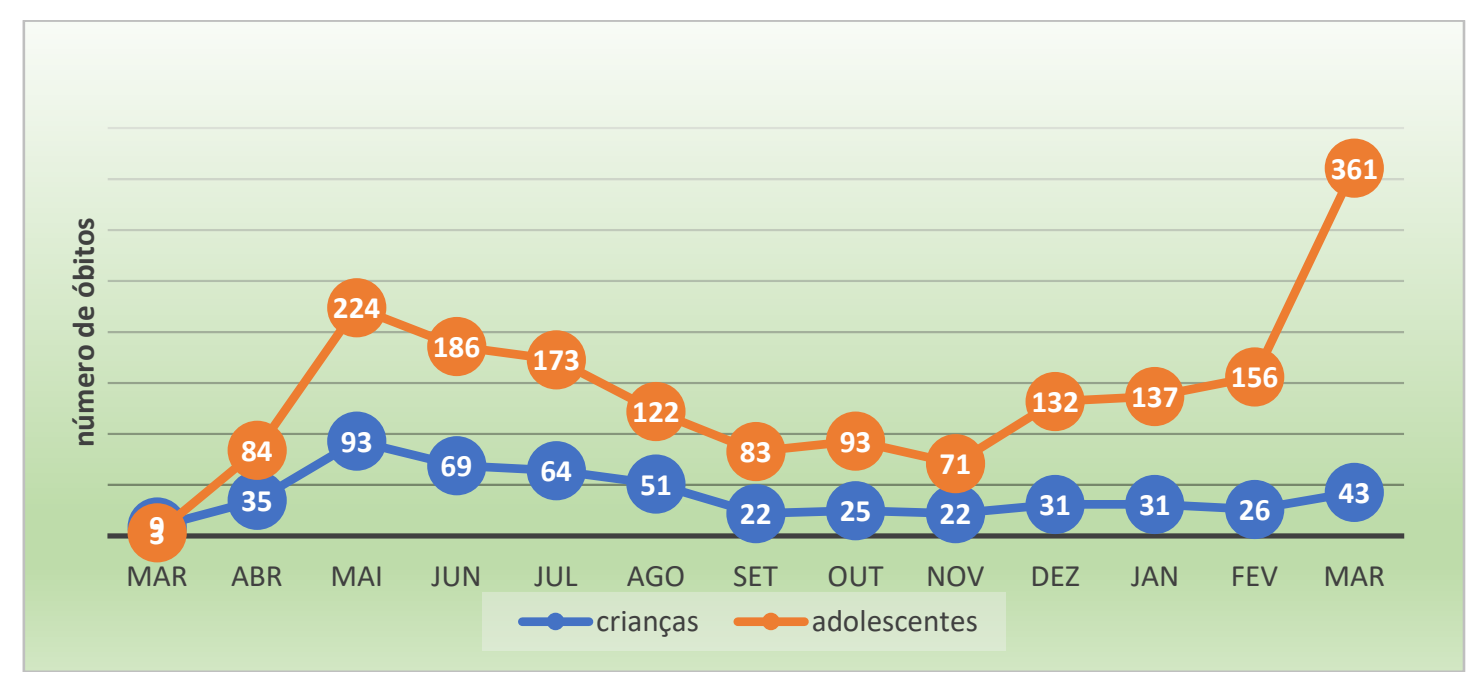

Fonte: elaboração própria a partir de https://transparencia.registrocivil.org.br/especial-covid

Figura 1 - Número de óbitos por COVID-19 em crianças e adolescentes segundo faixa etária e mês de notificação. Brasil, 01/03/2020 a 31/03/2021. 


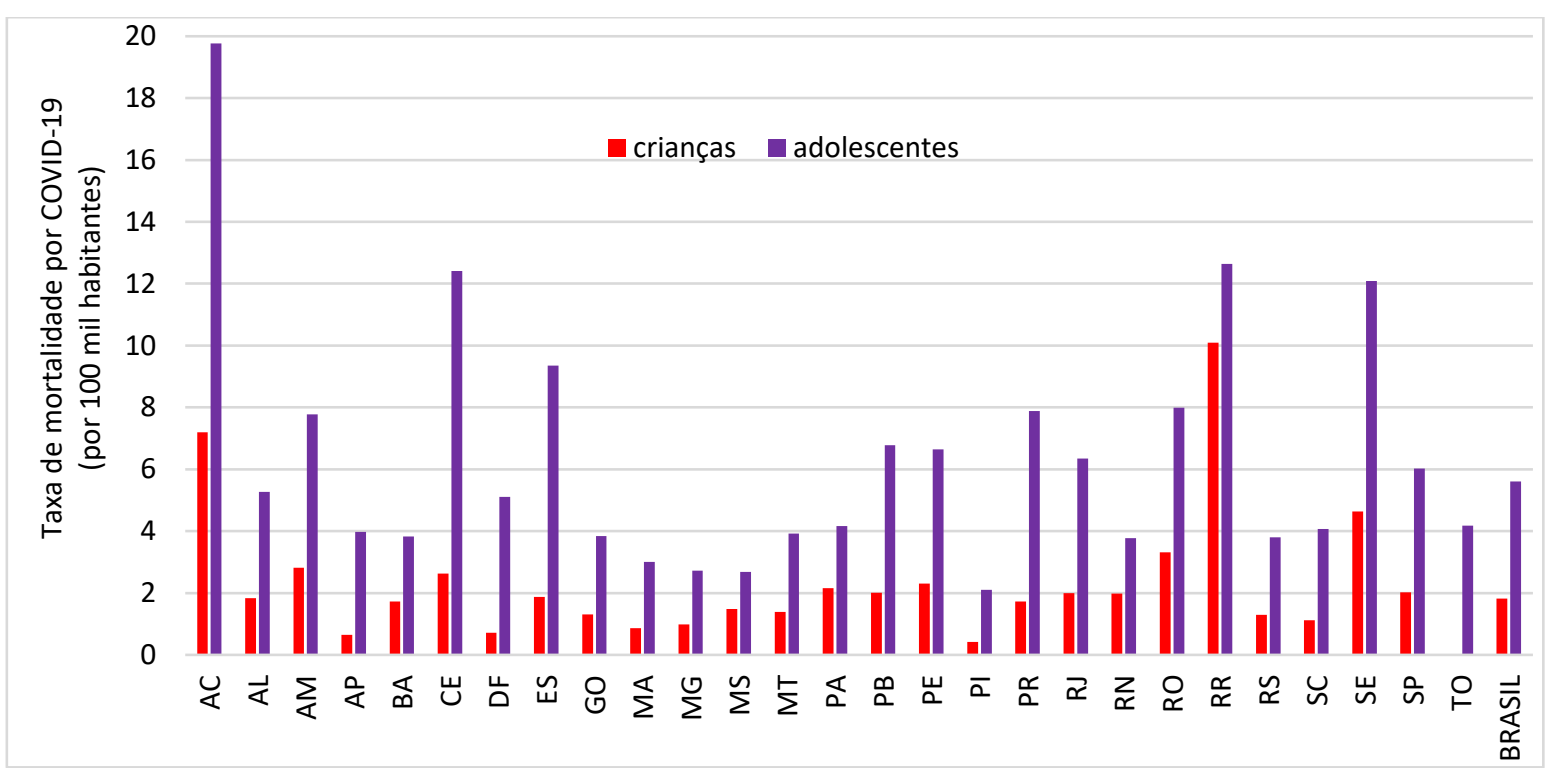

Fonte: elaboração própria a partir de: https://transparencia.registrocivil.org.br/especial-covid; http://tabnet.datasus.gov.br/cgi/tabcgi.exe?ibge/cnv/projpopuf.def

Figura 2 - Taxa de mortalidade por COVID-19 em crianças e adolescentes, por 100 mil habitantes, conforme Unidade da Federação, Brasil, 01/03/2020 a 31/03/2021. 
Tabela 1 - Casos e óbitos por Síndrome Inflamatória Multissistêmica Pediátrica temporalmente associada à COVID-19, notificados em crianças e adolescentes, segundo Unidade da Federação de notificação, Brasil 01/03/2020 a 31/03/2021.

\begin{tabular}{|c|c|c|c|c|c|}
\hline \multirow{3}{*}{$\begin{array}{l}\text { Unidade da } \\
\text { Federação a }\end{array}$} & \multicolumn{4}{|c|}{ Número acumulado } & \multirow{3}{*}{$\begin{array}{l}\text { Incidência de casos na } \\
\text { faixa etária 0-19 anos }\end{array}$} \\
\hline & \multicolumn{2}{|c|}{ Casos (idade) } & \multicolumn{2}{|c|}{ Óbitos (idade) } & \\
\hline & $(0-9)$ & $(10-19)$ & $(0-9)$ & $(10-19)$ & \\
\hline Acre $^{\mathbf{b}}$ & 1 & 1 & 1 & 0 & 1,2 \\
\hline Alagoas $\mathbf{b}$ & 20 & 8 & 0 & 1 & 3,6 \\
\hline Amazonas b & 22 & 0 & 3 & 0 & 2,6 \\
\hline Bahia $^{\mathbf{b}}$ & 50 & 15 & 3 & 0 & 1,7 \\
\hline Ceará b & 43 & 21 & 0 & 2 & 3,0 \\
\hline Distrito Federal $\mathbf{b}$ & 41 & 19 & 0 & 1 & 7,3 \\
\hline Espírito Santo b & 16 & 4 & 0 & 0 & 1,9 \\
\hline Goiás ${ }^{\mathbf{b}}$ & 17 & 3 & 0 & 1 & 1,1 \\
\hline Maranhão b & 7 & 3 & 0 & 0 & 0,7 \\
\hline Mato Grosso b & 5 & 3 & 0 & 0 & 0,9 \\
\hline Mato Grosso do Sul b & 0 & 1 & 0 & 1 & 0,1 \\
\hline Minas Gerais ${ }^{c}$ & 80 & 6 & 2 & 0 & 1,5 \\
\hline Pará b & 54 & 11 & 8 & 1 & 3,6 \\
\hline Paraíba ${ }^{\mathbf{b}}$ & 9 & 1 & 2 & 0 & 1,0 \\
\hline Paraná b & 17 & 8 & 1 & 2 & 0,8 \\
\hline Pernambuco ${ }^{\mathbf{b}}$ & 21 & 7 & 1 & 1 & 0,9 \\
\hline Piauí b & 7 & 2 & 1 & 0 & 1,2 \\
\hline Rio de Janeiro $^{\mathbf{b}}$ & 46 & 12 & 6 & 1 & 1,3 \\
\hline Rio Grande do Norte ${ }^{\mathbf{b}}$ & 8 & 4 & 0 & 0 & 1,4 \\
\hline Rio Grande do Sul d & 34 & 8 & 1 & 0 & 1,4 \\
\hline Rondônia ${ }^{b}$ & 1 & 1 & 0 & 0 & 0,3 \\
\hline Roraima ${ }^{\mathbf{b}}$ & 0 & 1 & 0 & 0 & 0,9 \\
\hline Santa Catarina ${ }^{\mathbf{b}}$ & 27 & 10 & 3 & 0 & 2,0 \\
\hline São Paulo b & 102 & 37 & 3 & 6 & 1,1 \\
\hline Sergipe ${ }^{b}$ & 4 & 1 & 0 & 0 & 0,7 \\
\hline Tocantins ${ }^{\mathbf{b}}$ & 2 & 1 & 0 & 0 & 0,8 \\
\hline Subtotal & 634 & 188 & 35 & 17 & \\
\hline BRASIL total & & 22 & & & 1,5 \\
\hline
\end{tabular}
a. Amapá sem casos e óbitos notificados
b. https://www.gov.br/saude/pt-br/media/pdf/2021/abril/08/boletim_epidemiologico_svs_12.pdf
c. https://coronavirus.saude.mg.gov.br/images/1 2021/01-boletim/07-04-Boletim Epidemiologico SIMP SE 13-2021.pdf
d. https://coronavirus.rs.gov.br/upload/arquivos/202103/26131529-boletim-epidemiologico-covid-19-coers-se-11.pdf 